\title{
Effectiveness of cognitive analytic therapy for personality disorders
}

Roger Mulder and Andrew M. Chanen

\section{Summary}

Personality disorders affect up to $50 \%$ of psychiatric out-patients. Most treatment studies have been performed in patients with borderline personality disorder. Structured psychosocial interventions for people with borderline personality disorders appear to have similar efficacy. There is some evidence that non-structured, non-specialised treatments offered by psychiatric general services might be ineffective and possibly harmful in patients with personality disorders. Cognitive analytic therapy is a time-limited, integrative psychotherapy, which appears to be effective for a range of personality disorders and superior to treatment as usual. Its practical nature and relatively short time limit may make it suitable for front-line clinical services.

\section{Declaration of interest}

None.
Roger Mulder (pictured) is a Professor in the Department of Psychological Medicine at the University of Otago, Christchurch. Andrew M. Chanen is an Associate Professor at the Orygen Youth Health Research Centre at the University of Melbourne and Clinical Director in the Orygen Youth Health Clinical Program at Northwestern Mental Health, Melbourne.

Personality disorders are a major cause of long-term functional disability $^{1}$ and they are among the most common disorders in clinical practice, affecting up to $50 \%$ of out-patients. ${ }^{2}$ However, the considerable achievements in the treatment of personality disorders over the past two decades have largely been confined to borderline personality disorder, particularly those individuals who engage in recurrent self-harm and have recurrent admissions to hospital. Moreover, the structured psychosocial interventions that have been developed for borderline personality disorder are mostly complex, time consuming and have very limited availability. When available, patients are given little or no choice about which treatment is offered and treatments are usually accessible only to those individuals who fulfil the twin criteria of severity of borderline personality disorder and motivation to seek and engage in care. Even then, non-completion rates for these interventions are often high. ${ }^{3}$

\section{Choosing between structured psychosocial interventions for personality disorder}

Clarke and colleagues ${ }^{4}$ in this issue report an important contribution to the literature on what to offer those patients who might not qualify for, or want, intensive borderline personality disorder treatment programmes and those with mixed personality pathology, while adding to the diversity of practical and relatively low-impact interventions suitable for use in front-line clinical services. There is an emerging consensus that structured psychosocial interventions, at least in the case of borderline personality disorder, are equivalent in treatment efficacy. ${ }^{5}$ Specialised treatments have been compared with alternative, well-structured general psychiatric interventions in several studies and produced non-existent or modest differences in outcomes. ${ }^{6-8}$ Clarke and colleagues' study has two unusual features. ${ }^{4}$ The first is that the participants had a range of

†See pp. 129-134, this issue. personality disorders, rather than just borderline personality disorder. The second is that their comparison treatment really was 'treatment as usual' and not an alternative structured intervention. The study therefore contrasts a specialised intervention - cognitive analytic therapy (CAT) - with a non-structured nonspecialised intervention, namely care from a community mental health team, clinical services and contacts with a general practitioner.

The results are interesting on two levels. First, a specialised structured treatment is effective with participants with a range of personality disorders other than borderline personality disorder. Whether this finding applies to other specialised treatments is unknown but, given the general equivalence of treatments for borderline personality disorder, it might. Second, treatment as usual appears to make some patients with personality disorders worse. Over half $(53 \%)$ of the treatment as usual participants met symptom criteria for more personality disorders by the end of the study. No participants in the CAT group deteriorated. If replicated, this finding has implications for general mental health services. It suggests that maintaining general interventions for personality disorders in mental health services is untenable and some type of specific structured intervention should be offered to all patients with significant personality disorder symptomatology. It reinforces the idea that 'treatment as usual' ranges from untested specialised treatments through to mutually hostile clinical contact with likely iatrogenic harm. ${ }^{6}$

Given that most structured, but apparently theoretically contrasting, interventions achieve similar outcomes, which one might general mental health services choose? Bateman has argued that all therapies share certain qualities: encouragement of increased activity and self-agency, a focus on emotional processing and increasing cognitive coherence, a structured manual and a validation of an active stance by the therapist. ${ }^{5}$ In front-line clinical services it might be argued that the choice of intervention, given it contains the qualities mentioned above, should reflect the realities of the workplace. Issues to consider include the practical nature of the treatment, its length and complexity, the ease of obtaining training and supervision, the cost, the treatment's efficacy with co-occurring mental disorders and importantly, patient preference.

\section{Cognitive analytic therapy}

Cognitive analytic therapy is a time-limited, integrative psychotherapy that has been developed over the past three decades 
by Anthony Ryle and subsequently others. ${ }^{9}$ However, the evidence base for CAT is small ${ }^{6}$ and has not kept up with its popularity in the UK, Finland and internationally. It arose from a theoretical and practical integration of elements of psychoanalytic object relations theory and cognitive psychology, subsequently developing into an integrated model of development and psychopathology. The self is seen in CAT to be fundamentally relational and social and characterised by an 'internalised' repertoire of relationship patterns (known as 'reciprocal roles'), acquired throughout early and subsequent development. These patterns form the basis of subsequent interpersonal relationships and self-management and are sustained by repetition. Developmental trauma and/or deprivation results in an internalised repertoire of harsh, restricted and/or inflexible relationship patterns, which are manifest as personality disorder.

Cognitive analytic therapy is practical and collaborative in style, with a particular focus upon understanding the individual's problematic relationship patterns and the thoughts, feelings and behavioural responses that result from these patterns. A central feature in CAT is the joint (patient-therapist) creation of a shared understanding of the patient's difficulties and their developmental origins, using plain-language written and diagrammatic 'reformulations'. These form the basis for understanding relationship problems both outside and within therapy, assist the patient to recognise and revise their dysfunctional relationship patterns and assist the therapist to avoid colluding (or recover from collusion) with such relationship patterns. Because of its strong relational focus, CAT has particular applicability to personality disorders, as it targets interpersonal and intrapsychic processes common to all personality disorders. Its integrative approach also encompasses co-occurring mental state disorders that are the norm in individuals with personality disorders presenting to front-line clinical services.

In personality disorder treatment, the perfect can often be the enemy of the good, with specialised interventions usually being prolonged and intensive at the cost of accessibility. A feature and strength of the CAT approach to individual therapy is its focus on being time limited (typically 16-24 weeks) and managing the ending in therapy from the outset. A 'good enough' ending is seen as an important therapeutic experience and a means of avoiding prolonged and/or collusive relationships. It also has a practical aim of improving access to therapy by increasing throughput in programmes. This may make it suitable for more widespread use in publically funded mental health systems and complementary to more prolonged and intensive interventions, which might be reserved either for those individuals with unsatisfactory outcomes from a trial of time-limited intervention or (in Clarke et al's study ${ }^{4}$ ) those with severe self-harm. Although this seems clinically sensible there is no evidence, at present, about the effectiveness of this approach.

Similar to other interventions, including longer-term ones, typical training programmes to become an independent CAT practitioner take at least 2 years. Although necessary, such requirements are likely to limit the uptake of such interventions across mental health systems. This underscores the need for simpler and more easily disseminated interventions, if that is possible to achieve, for problems that by their very nature are complex. It remains untested whether intervention at the service system level, such as teaching the basic principles and relational skills underlying structured interventions such as CAT to a broad range of clinicians, might change their interactions with patients with personality disorders to the extent that at least clinicians and services will first do no harm.

\section{Funding}

Orygen Youth Health Research Centre is funded by an unrestricted philanthropic grant from the Colonial Foundation, Melbourne, Australia.

Roger Mulder, MB ChB, PhD, FRANZCP, Department of Psychological Medicine, University of Otago, Christchurch; Andrew M. Chanen, MBBS, MPH, FRANZCP, Orygen Youth Health Research Centre, Centre for Youth Mental Health, The University of Melbourne and Orygen Youth Health Clinical Program, Northwestern Menta Health, Melbourne, Australia

Correspondence: Roger Mulder, University of Otago, Christchurch, PO Box 4345, Christchurch 8140, New Zealand. Email: roger.mulder@otago.ac.nz

First received 10 Jul 2012, final revision 28 Nov 2012, accepted 3 Dec 2012

\section{References}

1 Skodol AWE, Johnson JG, Cohen P, Sneed JR, Crawford TN. Personality disorder and impaired functioning from adolescence to adulthood. $\mathrm{Br} J$ Psychiatry 2007; 190: 415-20.

2 Zimmerman $\mathrm{M}$, Chelminski I, Young $\mathrm{D}$. The frequency of personality disorders in psychiatric patients. Psychiatr Clin North Am 2008; 31: 405-20.

3 Barnicot K, Katsakou C, Marougka S, Priebe S. Treatment completion in psychotherapy for borderline personality disorder: a systematic review and meta-analysis. Acta Psychiatr Scand 2011; 123: 327-38.

4 Clarke $S$, Thomas $P$, James $K$. Cognitive analytic therapy for personality disorder: randomised controlled trial. Br J Psychiatry 2013; 202: 129-34.

5 Bateman AW. Treating borderline personality disorder in clinical practice. Am J Psychiatry 2012; 169: 560-3.

6 Chanen AM, Jackson HJ, McCutcheon LK, Jovev M, Dudgeon P, Yuen HP, et al. Early intervention for adolescents with borderline personality disorder using cognitive analytic therapy: randomised controlled trial. Br J Psychiatry 2008; 193: 477-84.

7 Bateman A, Fonagy P. Randomized controlled trial of outpatient mentalization-based treatment versus structured clinical management for borderline personality disorder. Am J Psychiatry 2009; 166: 1355-64.

8 Clarkin JF, Levy KN, Lenzenweger MF, Kernberg OF. Evaluating three treatments for borderline personality disorder: a multiwave study. Am J Psychiatry 2007; 164: 922-8.

9 Ryle A, Kerr IB. Introducing Cognitive Analytic Therapy. John Wiley \& Sons, 2002. 